\title{
Obesidad y sedentarismo en niños de 4 años que asisten a dos jardines de infantes de la ciudad de Río Grande, Tierra del Fuego, Argentina
}

\author{
Obesity and sedentary lifestyles in four-years old children \\ attending two pre-schools in the city of Rio Grande, \\ Tierra del Fuego, Argentina
}

Dra. Ileana Ruth Berghtein ${ }^{a}$

\section{RESUMEN}

El inicio precoz del rebote adipocitario, y el sedentarismo, aumentan el riesgo de obesidad y sus comorbilidades en edades posteriores.

Río Grande se encuentra al noreste de Tierra del Fuego; su temperatura media mensual es la más baja de Argentina. Tiene la mayor proporción de población infantil de Argentina. Según la Encuesta Nacional de Nutrición y Salud de 2006, la prevalencia de obesidad en menores de 6 años es una de las más altas del país.

El objetivo de este estudio fue determinar la prevalencia de obesidad y sedentarismo en niños de 4 años de 2 jardines de infantes de esta ciudad. Se midió peso y talla. Se calculó índice de masa corporal y perímetro de cintura. Se evaluó con un cuestionario estructurado el tiempo semanal de actividades sedentarias.

El 27,3\% de los niños presentó sobrepeso y el $18,2 \%$, obesidad. El $23,6 \%$ tuvo circunferencia de cintura en percentil $\geq 90$, y el $70,1 \%$ fue considerado como sedentario.

El sedentarismo y el patrón de distribución grasa alertan sobre la necesidad de detectar factores de riesgo para síndrome metabólico en los grupos vulnerables.

Palabras clave: infancia temprana, obesidad, sobrepeso, sedentarismo.

a. Centro de

Rendimiento

Deportivo.

Consultorios

Médicos OSDIPP.

Río Grande,

Tierra del Fuego.

Correspondencia:

Dra. Ileana Ruth

Berghtein: ileana99@

gmail.com

Conflicto de intereses:

Ninguno que declarar.

Recibido: 6-9-2013

Aceptado: 16-6-2014

\section{ABSTRACT}

The precocious start of the adipocyte rebound, such as sedentarism, increases the risk of developing obesity and its comorbidities at later ages.

The city of Río Grande is located in the Northwestern part of Tierra del Fuego and its monthly average temperature is the lowest in all of Argentina. It also possesses the largest juvenile population (in proportion to the total) as well as a very low mortality rate. According to the 2006 National Health and Nutrition Survey, the prevalence of obesity among its children was also one of the highest in the country.

The objective of this investigation was to determine the prevalence of obesity and sedentarism in 4-year-old children; the sample was taken from two local kindergartens.

In order to achieve this, the children were weighed and measured, their body mass index and waist perimeter, as well as their percentiles, were calculated. A structured questionnaire was applied to characterize the average weekly time allotted to sedentary activities.

In this study, $27.3 \%$ of children were deemed to be overweight, while $18.2 \%$ were classified as obese. Furthermore, $23.6 \%$ presented a waist circumference in the $\geq 90$ percentile or higher and $70.1 \%$ fell in the sedentary classification. Both the predominance of sedentarism and the pattern of fat distribution are a clear alert towards the need to deepen the search for metabolic-risk syndrome factors in vulnerable groups.

Key words: early childhood, obesity, overweightness, sedentarism.

http:/ /dx.doi.org/10.5546/aap.2014.557

\section{INTRODUCCIÓN}

El Grupo Internacional de Trabajo para la Obesidad (IOTF) y la Organización Mundial de la Salud (OMS) han calificado la obesidad como la epidemia del siglo XXI por las dimensiones adquiridas a lo largo de las últimas décadas, su impacto sobre la morbimortalidad, la calidad de vida y el gasto sanitario. ${ }^{1}$

Un gran número de trabajos han generado un especial interés en el concepto de "períodos críticos", interpretados como etapas del desarrollo en las cuales alteraciones fisiológicas incrementan el riesgo de condiciones adversas en la salud futura.

Estos períodos serían el primer y tercer trimestre del embarazo, infancia temprana, etapa del rebote adipocitario (de 4 a 8 años de edad) y la adolescencia.

Los patrones genéticos de la huma- 
nidad no han sufrido cambios, pero sí los factores ambientales. ${ }^{2,3}$ Los niños son inherentemente activos si las condiciones ambientales lo permiten, tanto en lo referido a actividades no estructuradas como en la educación física y el deporte. Sin embargo, una investigación reciente muestra que las actividades sedentarias más comunes (videojuegos, televisión) llegan a ocupar cuatro horas diarias en promedio. La mayor parte de estas ocurre en el ámbito del hogar.

Así mismo, el espacio físico y la disponibilidad de elementos para el desarrollo de actividades deportivas se correlacionan con el número de horas de actividad física, sobre todo en adolescentes. ${ }^{3}$

La escuela como ambiente físico, social y cultural juega también un papel importante, que influye en la educación, sistema de creencias, comportamientos y hábitos de los niños que allí asisten. $^{4}$

Río Grande se encuentra al noreste de Tierra del Fuego y tiene la menor temperatura media mensual de Argentina. Tiene la mayor proporción de población infantil y la mortalidad infantil se encuentra entre las más bajas del país. ${ }^{5}$ Sin embargo, la Encuesta Nacional de Nutrición y Salud (ENNyS), que provee datos nacionales, en el grupo de niños menores de 6 años, mostró una prevalencia de obesidad del 10,4\% y aclaró que la provincia de Tierra del Fuego se encontraba entre las que tenían prevalencia mayor. ${ }^{6}$

El objetivo de este trabajo es determinar la prevalencia de obesidad y sedentarismo en niños de 4 años de dos jardines de infantes de esta ciudad.

\section{POBLACIÓN Y MÉTODOS}

En la búsqueda de una población representativa de los distintos sectores de la ciudad, se eligieron para el estudio los dos jardines de infantes que albergan más número de salas y que, por su ubicación céntrica, reciben niños de distintos barrios de la ciudad.

De acuerdo con la consulta realizada en la Dirección de Supervisión Escolar, en el momento del estudio (setiembre y octubre de 2012), la matrícula para sala de 4 años en la ciudad fue de 1220 niños.

Se pidió autorización para realizar el estudio a las direcciones de los jardines y posteriormente a los padres. Se consideraron como criterios de inclusión los siguientes: niños de 4 años hasta 4 años y 11 meses cumplidos, cuyos padres dieran consentimiento para participar del estudio. El criterio de exclusión fue los niños que hubieran estado enfermos en los últimos 7 días.

Los niños fueron pesados y medidos sin calzado, vestidos, sin guardapolvo y con ropa ligera, todos por el mismo operador.

Para las mediciones, se utilizó una balanza mecánica marca CAM, un tallímetro portátil marca Nel y una cinta métrica extensible.

Se calculó y clasificó el IMC y el perímetro de cintura, según normas del último Consenso de diagnóstico de sobrepeso y obesidad de la Sociedad Argentina de Pediatría. ${ }^{7}$

Para percentiles de perímetro de cintura, fueron tomados de referencia los valores de Fernández y cols. ${ }^{8,9}$

Para caracterizar en promedio el tiempo semanal de actividades sedentarias, considerando principalmente el tiempo de exposición a pantallas (televisión, videojuegos, computadora), se utilizó un cuestionario estructurado (test corto de actividad física Krece-plus), dirigido a los padres de los niños estudiados, que permitió un cribado rápido del nivel de actividad/ inactividad ${ }^{10}$ (Anexo).

Este test puntúa positivamente la actividad física realizada en tiempo libre y, de manera negativa, las horas dedicadas a la televisión, videojuegos y computadora. Según la puntuación, el estilo de vida del niño se clasificó como malo (5 o menos), regular (de 6 a 8 ) o bueno (mayor de 8$){ }^{11}$

\section{Análisis estadístico}

Se utilizó el programa estadístico STATA 9.1.

Las variables cualitativas se expresaron como proporción (porcentaje) y, para la prevalencia de sobrepeso/obesidad, se calculó, además, el IC $95 \%$.

\section{RESULTADOS}

De los 122 niños que asisten a las salas de 4 seleccionadas, se analizaron los datos provenientes de 110 niños. De los 12 niños restantes, 3 de ellos no fueron autorizados a participar del trabajo, y 9 no estaban presentes el día establecido para los controles.

Sesenta de 110 niños (54,5\%) poseían peso normal o bajo; mientras que 30 (27,3\%) tenían sobrepeso y $20(18,2 \%)$, obesidad. Por lo tanto, la proporción de niños con sobrepeso/obesidad en la muestra estudiada fue del 45,5\% (IC 95\%: $36,0-54,9 \%)$.

De los 107 niños cuyos padres respondieron la encuesta sobre actividad física, solo 3 niños $(2,8 \%)$ 
obtuvieron buena puntuación, mientras que 29 $(27,1 \%)$ tuvieron una puntuación regular y 75 $(70,1 \%)$ lograron una puntuación mala.

Entre los 75 niños sedentarios, hubo 34 con sobrepeso/obesidad (45,3\%), mientras que, entre los 32 no sedentarios, hubo $13(40,6 \%)$. Si bien hubo mayor proporción de niños con sobrepeso y obesidad entre los sedentarios, esta no fue estadísticamente significativa.

Veintiséis niños $(23,6 \%)$ presentaban circunferencia de cintura igual o mayor a 90 , considerado indicador de riesgo para el desarrollo de síndrome metabólico.

\section{DISCUSIÓN}

Los factores que intervienen en los procesos de crecimiento y desarrollo son numerosos y actúan de manera coordinada e influenciados por factores del medioambiente, que determinarán su resultado final.

La literatura acerca del tema en países tan dispares en desarrollo y climas, como Canadá, Australia, Gran Bretaña, Estados Unidos, China, México, Portugal, etc., coincide tanto en la prevalencia como en el análisis de sus múltiples causas y su impacto en la salud pública, y propone poner más énfasis en la actividad física escolar y hacer de ella una actividad prioritaria junto con la promoción de otros hábitos saludables. ${ }^{4}$

En Argentina, se han desarrollado, durante las últimas décadas, diversos estudios de prevalencia, aunque con metodologías y criterios diagnósticos distintos, pero todos concluyen que, en el país, la obesidad es la enfermedad nutricional más prevalente, de comienzo a temprana edad. Además, de acuerdo con la información provista por la Encuesta Nacional de Factores de Riesgo 2009 (ENFR), el 54,9\% de la población es inactiva. ${ }^{12}$

En particular en nuestra ciudad, que presenta la menor temperatura media mensual de Argentina, la estructura demográfica creció como consecuencia del más rápido proceso de migración interna del país y está conformada por habitantes provenientes de todo el territorio. Ellos debieron adaptarse a una zona donde la temperatura promedio en enero es de $11^{\circ} \mathrm{C}, \mathrm{y}$ la temperatura promedio de julio es de $-0,3$, lo cual podría influir en las posibilidades o el tiempo en el que los niños realizan juegos al aire libre. En este estudio, menos del 3\% de los niños presentaron un nivel adecuado de actividad física. Si bien se ha utilizado un test que no había sido previamente validado en este medio, cabe destacar que este ha sido aplicado en otras poblaciones de habla castellana con niños de la misma edad y no utiliza términos que no sean de uso corriente en nuestro medio. . $^{10,11}$

Comparando con los datos de la Encuesta Nacional de Salud y Nutrición 2006, habría una tendencia en ascenso del sobrepeso y obesidad en estos niños que coincide con las proyecciones de la OMS.

Los patrones de desarrollo socioeconómico, a partir de la ley de promoción industrial en la provincia, el aumento de la disponibilidad de alimentos y bebidas industrializadas, el exponencial desarrollo de tecnologías de la información, la comunicación y el conocimiento han tenido una decisiva influencia en los cambios de sistemas de creencias y en la homogeneización y aceptación sin resistencias de hábitos y modelos de vida obesogénicos.

En nuestro país, no conocemos con exactitud cuáles son las cifras de sedentarismo en la infancia. Sabemos que los avances tecnológicos propiciaron la disminución de la actividad física en la población a nivel mundial. Estas cifras marcan tendencias generales, pero es indudable que hay factores que determinan un mayor riesgo de sedentarismo en la población infantil. ${ }^{13}$

La infancia temprana (período preescolar) es un momento importante para la incorporación de hábitos saludables, así como para la identificación temprana de conductas de riesgo orientadas a la prevención de sobrepeso y obesidad.

Además, existe evidencia de que la educación física escolar, por su condición de requerir asistencia obligatoria, con presencia de profesionales y un ambiente seguro, permite alcanzar las recomendaciones establecidas como necesarias para cada edad, así como estimular la actividad física más allá de la escuela. ${ }^{4,14}$

En el caso de la provincia de Tierra del Fuego, y su condición climática, tal vez esta sea la única oportunidad para estimular el placer y necesidad de movimiento en los niños.

Es por ello por lo que, a pesar de ser una muestra pequeña y por conveniencia, los datos encontrados alertan sobre la necesidad de estudiar en mayor profundidad esta problemática y proponer medidas que resulten en una mejora tanto de los hábitos alimentarios como de la actividad física.

\section{CONCLUSIONES}

En la ciudad de Río Grande, en la población estudiada, el 45,5\% de los niños presenta exceso de peso. Es evidente también el predominio de 
sedentarismo $(70,1 \%)$ tanto en los grupos con sobrepeso como en el de peso normal.

El patrón de distribución grasa alerta sobre la necesidad de profundizar la búsqueda de factores de riesgo para síndrome metabólico en grupos vulnerables.

\section{REFERENCIAS}

1. Resolución WHA57.17. Estrategia Mundial sobre Régimen Alimentario, Actividad Física y Salud. En 57 ${ }^{\mathrm{a}}$ Asamblea Mundial de la Salud, Ginebra, 17-22 de mayo del 2004. Resoluciones y decisiones, anexos. Ginebra: Organización Mundial de la Salud; 2004.

2. KovalskysI, Bay L, RauschHerscoviciC, BernerE. Prevalencia de obesidad en una población de 10 a 19 años en la consulta pediátrica. Arch Argen Pediatr 2003;101(6):441-7.

3. ILSI Argentina. Comité de nutrición, obesidad y actividad física. Análisis de los factores vinculados a sobrepeso y obesidad en niños de 10 y 11 años que asisten a escuelas públicas en el área metropolitana de Buenos Aires. Buenos Aires: ILSI Argentina; 2007.

4. Dobbins M, Husson H, De Corby K, LaRocca RL. Schoolbased physical activity programs for promoting physical activity and fitness in children and adolescents aged 6 to 18. Cochrane Database Syst Rev 2013;2:CD007651.

5. Instituto Nacional de Estadística y Censos (INDEC). Censo Nacional de población, hogares y viviendas 2010. Resultados definitivos. Serie $\mathrm{B} \mathrm{N}^{\circ}$ 2. Buenos Aires: INDEC; 2012.

6. Argentina. Ministerio deSalud. Encuesta Nacional deNutrición y Salud. Documento de resultados 2007. Buenos Aires: MinisteriodeSalud;2007. Disponibleen:http://www.msal. gov.ar/images/stories/bes/graficos/0000000257cnt-a08ennys-documento-de-resultados-2007.pdf. [Acceso: 16 de junio de 2014].

7. Comité Nacional de Nutrición. Guías de práctica clínica para la prevención, el diagnóstico y el tratamiento de la obesidad. Arch Argent Pediatr 2011;109(3):256-66.

8. Sociedad Argentina de Pediatría. Comité Nacional de Crecimiento y Desarrollo. Guías para la Evaluación del Crecimiento. Buenos Aires: Sociedad Argentina de Pediatría; 2001.

9. Hirschler V, Delfino AM, Clemente G, Aranda C, et al. ¿Es la circunferencia de cintura un componente del síndrome metabólico en la infancia? Arch Argent Pediatr 2005;103(1):7-13.

10. Serra Majem L, Aranceta Bartrina J, Ribas Barba L, Sangil Monroy $\mathrm{M}$, et al. Crecimiento y desarrollo: dimensión alimentaria y nutricional. En: Serra Majem L, Aranceta Bartrina J, Rodríguez Santos F, eds. Crecimiento y desarrollo: Estudio en Kid. Barcelona: Masson; 2003. Págs.45-55.

11. MorenoEsteban B, Charro Salgado AL. Nutrición, actividad física y prevención de la obesidad: estrategia NAOS. Madrid: Panamericana; 2006.

12. Ferrante D, Linetzky B, Konfino J, King A, et al. Encuesta nacional de factores de riesgo 2009: evolución de la epidemia de enfermedades crónicas no transmisibles en Argentina. Estudio de corte transversal. Rev Argent Salud Publ 2011;2(6):34-41.

13. Metallinos-Katsaras ES, Freedson PS, Fulton JE, Sherry B. The association between an objective measure of physical activity and weight status in preschoolers. Obesity (Silver Spring) 2007;15(3):686-94.

14. Collings PJ, BrageS, Ridgway CL, Harvey NC, etal.Physical activity intensity, sedentary time, and body composition in preschoolers. Am J Clin Nutr 2013;97(5):1020-8. 


\section{Anexo}

\section{Test corto de actividad física adaptado de Krece-plus}

Escuela:

Curso:

Turno:

Nombre y apellido:

Fecha de nacimiento:

Queremos conocer cuál fue el nivel de actividad física de tu hijo en los últimos 7 días (última semana). Esto incluye todas aquellas actividades, como deportes, gimnasia o danza, que hacen transpirar o sentirse cansado, o juegos que hagan que se acelere su respiración, como jugar, saltar, correr, trepar y otras.

\section{Recuerda:}

1. No hay preguntas buenas o malas. Esto NO es un examen.

2. Contesta las preguntas de la forma más honesta y sincera posible. Esto es muy importante.

¿Estuvo enfermo esta última semana o algo impidió que hiciera normalmente actividades físicas?

Sí.

No.

¿Cuántas horas por día ve televisión, juega videojuegos y usa la computadora?

(Si hace las 3 cosas, sumá todas las horas; esa será la respuesta).

0 horas.

1 hora.

2 horas.

3 horas.

4 horas.

5 horas.

Fuera de la escuela, ¿cuántas horas dedica a hacer deportes o danzas o jugar a juegos en los que se corre, salta o se mueve intensamente? (Intensamente significa que, al moverse, transpira, se agita o siente que el corazón late más fuerte).

0 horas.

1 hora.

2 horas.

3 horas.

4 horas.

5 horas. 